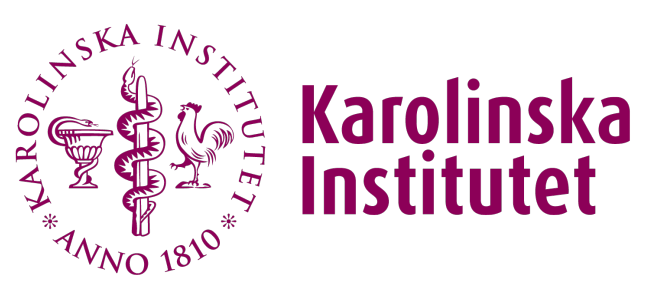

Karolinska Institutet

http://openarchive.ki.se

This is a Peer Reviewed Accepted version of the following article, accepted for publication in Behavior genetics.

\title{
A family-based study of the association between labor induction and offspring attention-deficit hyperactivity disorder and low academic achievement
}

Wiggs, Kelsey K; Rickert, Martin E; Hernandez-Diaz, Sonia; Bateman, Brian T; Almqvist, Catarina; Larsson, Henrik; Lichtenstein, Paul; Öberg, Anna Sara; D'Onofrio, Brian M

Behav Genet. 2017 Jul;47(4):383-393.

http://doi.org/10.1007/s10519-017-9852-4

http://hdl.handle.net/10616/45978

If not otherwise stated by the Publisher's Terms and conditions, the manuscript is deposited under the terms of the Creative Commons Attribution-NonCommercial-NoDerivatives License (http://creativecommons.org/licenses/by-nc-nd/4.0/), which permits non-commercial re-use, distribution, and reproduction in any medium, provided the original work is properly cited, and is not altered, transformed, or built upon in any way. 


\section{(19) \\ Karolinska Institutet}

This is the peer reviewed version of the following article Behav Genet. 2017 Jul;47(4):383-393. which has been published in final form at

http://dx.doi.org/10.1007/s10519-017-9852-4

A family-based study of the association between labor induction and offspring attention-deficit hyperactivity disorder and low academic achievement.

Wiggs, K.K.; Rickert, M.E.; Hernandez-Diaz, S.; Bateman, B.T.; Almqvist, C.; Larsson, H.; Lichtenstein, P.; Öberg, A.S.; D'Onofrio, B.M.

Access to the published version may require subscription. Published with permission from: Springer Nature 


\section{A family-based study of the association between labor induction and offspring} attention-deficit hyperactivity disorder and low academic achievement

Kelsey K. Wiggs ${ }^{\mathrm{a}}$

Martin E. Rickert ${ }^{\mathrm{a}}$

Sonia Hernandez-Diaz ${ }^{\mathrm{b}}$

Brian T. Bateman ${ }^{\mathrm{c}, \mathrm{d}}$

Catarina Almqvist ${ }^{\mathrm{e}, \mathrm{f}}$

Henrik Larsson ${ }^{\mathrm{e}, \mathrm{g}}$

Paul Lichtenstein ${ }^{\mathrm{e}}$

Anna Sara Oberg ${ }^{\mathrm{b}, \mathrm{e}}$

Brian M. D’Onofrio ${ }^{\mathrm{a}}$

${ }^{\mathrm{a}}$ Department of Psychological and Brain Sciences, Indiana University, Bloomington, IN, USA

${ }^{\mathrm{b}}$ Department of Epidemiology, Harvard T.H. Chan School of Public Health, Boston, USA

${ }^{\mathrm{c}}$ Division of Pharmacoepidemiology and Pharmacoeconomics, Department of Medicine at Brigham and Women's Hospital, Harvard Medical School, Boston, USA

${ }^{\mathrm{d} D e p a r t m e n t ~ o f ~ A n e s t h e s i a, ~ C r i t i c a l ~ C a r e, ~ a n d ~ P a i n ~ M e d i c i n e ~ a t ~ M a s s a c h u s e t t s ~ G e n e r a l ~ H o s p i t a l, ~}$ Harvard Medical School, Boston, USA

${ }^{\mathrm{e}}$ Department of Medical Epidemiology and Biostatistics, Karolinska Institutet, Stockholm, Sweden

${ }^{\mathrm{f}}$ Pediatric Allergy and Pulmonology Unit at Astrid Lindgren Children's Hospital, Karolinska University Hospital, Karolinska Institutet, Stockholm, Sweden

${ }^{\text {g}}$ School of Medical Sciences, Orebro University, Orebro, Sweden 
All correspondence should be sent to:

\author{
Kelsey K. Wiggs \\ Department of Psychological and Brain Sciences \\ Indiana University \\ 1101 E. 10th St. Bloomington, IN 47405
}

Phone: 812-856-0843

Fax: 812-856-4544

kkwiggs@indiana.edu

\title{
CONFLICTS OF INTEREST
}

H Larsson has served as a speaker for Eli-Lilly and Shire and has received research grants from Shire; all outside the submitted work. Paul Lichtenstein has served as a speaker for Medice. All other authors report no conflicts of interest. 


\begin{abstract}
The current study examined associations between labor induction and both (1) offspring attention-deficit hyperactivity disorder (ADHD) diagnosis in a Swedish birth cohort born 1992$2005(\mathrm{n}=1,085,008)$ and (2) indices of offspring low academic achievement in a sub-cohort born1992-1997 ( $\mathrm{n}=489,196)$. Associations were examined in the entire sample (i.e. related and unrelated individuals) with adjustment for measured covariates and, in order to account for unmeasured confounders shared within families, within differentially exposed cousins and siblings. We observed an association between labor induction and offspring ADHD diagnosis and low academic achievement in the population. However, these associations were fully attenuated after adjusting for measured covariates and unmeasured factors that cousins and siblings share. The results suggest that observed associations between labor induction and ADHD and low academic achievement may be due to genetic and/or shared environmental factors that influence both mothers' risk of labor induction and offspring neurodevelopment.
\end{abstract}

\title{
KEYWORDS
}

Labor Induction• Attention-Deficit Hyperactivity Disorder • Academic Achievement $\bullet$ Family Studies • Sibling Comparison• Cousin Comparison WORD COUNT Abstract (149/150) • Manuscript (4,377) • Tables (11) • Figures (2) 


\section{INTRODUCTION}

The Developmental Origins of Health and Disease (DOHaD) hypothesis proposes that early life experiences are causally linked to the development of many physical, mental, and cognitive problems throughout the lifespan (e.g. Barker, 1998). Given that these problems have significant educational and economic implications in adulthood, greater efforts need to be made to identify early life prevention and intervention programs (Heckman, 2012).

An important example is birth following labor induction, which occurred in 23.3 percent of births in the U.S. in 2012 (Osterman \& Martin, 2014). Although labor induction often benefits both maternal health (e.g., induction for pre-eclampsia) and short term outcomes for the infant (Nassar, Adra, Chakhtoura, Gómez-Marín, \& Beydoun, 1998; Vivian-Taylor et al., 2011; Smith \& Merrill, 2006), recent studies have found that labor induction predicts long-term outcomes such as offspring autism spectrum disorder (ASD) (Gregory, Anthopolos, Osgood, Grotegut, \& Miranda, 2013) and attention-deficit hyperactivity disorder (ADHD) (Kurth \& Haussmann, 2011; Desiree Silva, Lyn Colvin, Erika Hagemann, \& Carol Bower, 2014) in offspring. This association has raised concern from the media, health professionals, and expecting mothers.

A causal effect of exposure to labor induction and offspring neurodevelopmental problems has at least two plausible mechanisms. One involves the method used to induce labor, as it has been suggested that administration of Pitocin (exogenous oxytocin) during labor could lead to downregulation of oxytocin receptors in the fetal brain (Wahl, 2004;Cochran, Fallon, Hill, \& Frazier, 2013), which in turn could result in an array of neurodevelopmental problems (including ADHD and ASD) (Gregory et al., 2013; Kurth \& Haussmann, 2011; Desiree Silva et al., 2014), Another plausible explanation is that the intervention to induce labor (irrespective of 
method) is causally linked to offspring neurodevelopmental problems by means of increased risk for other obstetric complications. For example, labor induction increases risk of fetal distress due to uterine hyper-stimulation (Nassar et al., 1998; Smith \& Merrill, 2006), which may have downstream effects on the fetal brain via hypoxia (Luthy, Malmgren, \& Zingheim, 2004; Smith \& Merrill, 2006; Spong et al., 2007). Additionally, labor induction is associated with instrumental delivery and epidural analgesia (Cammu, Martens, Ruyssinck, \& Amy, 2002).

However, the association between labor induction and offspring neurodevelopmental outcomes may also have non-causal explanations. For example, many pregnancy complications that increase maternal risk to be induced (e.g. pre-eclampsia) (Nassar et al., 1998) are also associated with neurodevelopmental outcomes (Walker et al, 2015; Getahun et al., 2013). Recently, Oberg et al. (2016) sought to test alternative explanations for the association between labor induction and ASD by comparing differentially exposed cousins and siblings, which allows control for unmeasured genetic and environmental factors that the relatives share. The results indicated that the risk of being diagnosed with ASD was similar in siblings where one was exposed to labor induction and the other was not. Other studies investigating the association between labor induction and neurodevelopmental outcomes may thus have been limited by lack of control for several potentially important confounders (Gregory et al., 2013; Kurth \& Haussmann, 2011; Desiree Silva et al., 2014). Our current understanding of the consequences of induction is also limited in size and number of studies, further suggesting that conclusions have been prematurely drawn (Saade, Sibai, \& Silver, 2014; Stitely, 2014; Vintzileos \& Ananth).

Furthermore, the scope of the association between labor induction and offspring neurodevelopment is unclear, as it is unknown if labor induction predicts individual differences in other areas likely impacted by neurodevelopmental function, such as offspring academic 
achievement. The current study sought to address these limitations of the existing literature by examining the association (if any) between labor induction and offspring ADHD diagnosis and low academic achievement in a Swedish cohort. Importantly, we examined the association in the population, as well as in differentially exposed cousins and siblings, which allowed adjustment of unmeasured factors that the family members share (D’Onofrio, Lahey, Turkheimer, \& Lichtenstein, 2013). An example of an environmental unmeasured confounding factor may be low socioeconomic status, often measured imperfectly as a covariate, which is associated with both medical conditions necessitating induction (e.g. gestational diabetes) (Kjos, Henry, Montoro, Buchanan, \& Mestman, 1993; Berkowitz, Lapinski, \& Lee, 1992; Nomura et al., 2012) and offspring ADHD (Nomura et al., 2012). Genetic factors also influence both a mother's risk for conditions requiring induction (e.g. gestational diabetes) (Zhang et al., 2013) and offspring ADHD (Larsson, Chang, D’Onofrio, \& Lichtenstein, 2014). The Swedish registers also have specific measures of maternal and pregnancy characteristics that we identified and included as measured covariates. Thus, this study adds to the current literature by providing a more rigorous evaluation of whether the association between labor induction and offspring ADHD and academic achievement is likely to be causal in nature.

\section{METHODS}

\section{DATA SOURCE}

Each individual in Sweden has their own unique registration number, which enables the tracking of people over time in several national registers (Ludvigsson, Otterblad-Olausson, Pettersson, \& Ekbom, 2009). The Multi-Generation Register (MGR) links all offspring to their parents (biological or adopted) (Statistics Sweden, 2010). This allows identification and linkage of siblings and cousins, as well as many other familial relations, throughout the Swedish 
population.

We used the Medical Birth Register (MBR), which contains information on all live births from 1973, including information on all antenatal visits, the delivery, and pediatric examination (Cnattingius, Ericson, Gunnarskog, \& Kallen, 1990; National Board of Health and Welfare, 2003). All diagnoses and procedures until discharge were also recorded using the World Health Organization's (WHO) International Classification of Diseases (ICD) code (WHO, 1992). The first antenatal visit typically occurs between weeks 8 and 12 and includes standardized reports of mothers' weight, height, cohabitation status (married vs. living alone), age, reproductive history, use of tobacco, and some chronic conditions (e.g. diabetes, renal disease). The MBR also has records on a number of obstetric measures (see below for more information). We also used the National Patient Register (NPR) with records of all inpatient psychiatric visits since 1973 and the majority of specialist outpatient visits since 2001 (National Board of Health and Welfare, 2009), and the Prescribed Drug Register (PDR), which provides information on all drugs dispensed in Sweden since July, 2005. Lastly, we used the National School Register to obtain school grades and standardized test scores for math, English, and Swedish ascertained in $9^{\text {th }}$ grade (age 15-16) and the Education Register for information on yearly assessments of the highest levels of education achieved.

\section{MEASURES}

We identified our exposure, labor induction, in the MBR by a yes/no check box on the delivery chart, available since 1991. From 1997 onward, it can also be identified via discharge record. Because check box indicators are considered more reliable than code reporting, check box records were used as our main measure for labor induction, and codes were used as a compliment (Frost, Starzyk, George, \& McLaughlin, 1984). 
Our outcomes of interest were an ADHD diagnosis, school grades, and standardized test scores in math, English, and Swedish ascertained in $9^{\text {th }}$ grade (age 15-16). We identified ADHD diagnoses from ICD-10 codes in the NPR (WHO, 2004), or from dispensation of ADHD medication the PDR, similar to previous studies (Chang et al., 2014; Chen et al., 2013; Larsson, Sariaslan, Långström, D'Onofrio, \& Lichtenstein, 2014; Skoglund, Chen, Lichtenstein, \& Larsson, 2014). Cases of ADHD were identified by specialist visits. Authority to prescribe medication is reserved for specialists. As such, although we were able to capture all ADHD cases requiring medication, it is possible that we did not capture cases that did not require medication. Overall grades were identified in the National School Register, pooled based on sixteen subjects and ranging from 0-320 in score, with a score below the mean indicating at least one failed class (Lambe, Hultman, Torrång, MacCabe, \& Cnattingius, 2006). Standardized test scores in math, English, and Swedish, also identified in the National School Register, were ordinal scores that have four levels between assessment years 1998 and 2012 and six levels from 2013 onward (8\% of our cohort). We standardized and converted all these variables to z-scores by year according to the population means. Next, we dichotomized all our academic variables using the lowest quartile as the threshold, similar to previous research (Bramson et al., 2016). Low academic achievement has also been documented to be associated with other outcomes, such as criminality (D’Onofrio et al., 2010).

We selected measured covariates for confounder adjustment based on known and observed associations between these variables and our exposure and outcomes of interest. They were identified in the MBR and the Education Register, and included maternal characteristics such as highest level of education, country of birth, and civil status, and pregnancy characteristics such as maternal age at delivery, birth year, parity, early pregnancy body mass 
index (BMI), smoking during pregnancy, gestational diabetes, gestational hypertension, preeclampsia, chorioamniontis, urogenital infection, high-risk pregnancy, premature rupture of membranes, post-term gestation, intrauterine growth restriction, and offspring sex.

\section{STUDY POPULATION}

We selected a birth cohort born 1992-2005 $(\mathrm{n}=1,362,950)$ to examine the association between labor induction and offspring ADHD (labor induction available since 1991 and follow up available up to the end of 2013). For the association with academic achievement this cohort was restricted to those born 1992-1997 $(n=628,622)$ because standardized tests occur at the age of 15-16 and individuals born after 1997 have not yet been assessed.

The majority of missing information stemmed from maternal early pregnancy BMI (16\%), civil status (6\%), and smoking during pregnancy (6\%). We excluded individuals with missing data on any covariates of interest (see Table I), and performed sensitivity analyses to investigate the potential influence of missing BMI. Our final sample was $n=1,085,008$ for the cohort born between 1992 and 2005 and $n=489,196$ for the restricted cohort born between 1992 and 1997. In the full cohort (1992-2005) we further identified 424,510 sibling pairs, 64,762 (15.3\%) of which were differentially exposed to labor induction, and 294,348 cousin pairs, $51,931(17.6 \%)$ of which were differentially exposed to labor induction. In the restricted cohort (1992-1997) we identified 102,460 sibling pairs, 12,129 (11.8\%) of which were differentially exposed, and 69,720 cousin pairs, 9,949 (14.3\%) of which were differentially exposed.

\section{DATA ANALYTIC STRATEGY}

We ran all analyses using SAS statistical software version 9.4. First, we examined the distributions of maternal and pregnancy characteristics overall and stratified by labor induction (presented in Table I). Second, we examined the associations (odds ratios) between labor 
induction and these selected covariates (presented in Table II). Third, we examined the distributions of maternal and pregnancy characteristics among siblings born 1992-2005 and belonging to families in which (1) at least one sibling had an ADHD diagnosis and (2) siblings without an ADHD diagnosis had censoring time greater than or equal to their sibling who received an ADHD diagnosis (presented in Table SI). We report the distributions among siblings meeting these criteria because they contribute to the estimate of the association between labor induction and offspring ADHD in the sibling comparisons. Fourth, we ran models examining the association between labor induction and our two sets of outcomes (i.e. ADHD and low academic achievement) of interest, which we present below separately by outcome type.

\section{Labor Induction to Offspring ADHD}

First, we plotted the association between labor induction and ADHD graphically in the population and in differentially exposed siblings using Kaplan-Meier estimation. Next, we used cox proportional hazard regression to examine the association, using age as underlying time scale and allowing censoring due to emigration, death, or end of follow up. Our model fitting procedure was similar to that which was used in Oberg et al. (2016).

\section{Labor Induction to Offspring Academic Achievement}

First, we examined the frequency of low grades and low standardized test scores in math, English, and Swedish in the population and in differentially exposed siblings (presented in Table III). Next, we fit logistic regression models to assess the association between labor induction and offspring low overall grades and low test scores in math, English, and Swedish.

\section{Labor Induction to all Offspring Outcomes}

First, we ran crude models to assess the associations between labor induction and offspring outcomes without adjustment. Second, we adjusted for offspring year of birth and 
parity, mother's age at childbearing, and maternal characteristics that aren't likely to vary meaningfully between births (i.e. stable maternal variables): highest level of education achieved, country of birth, and civil status. Third, we added adjustment for pregnancy characteristics that could be unique to each pregnancy (i.e. pregnancy specific variables), including offspring sex, maternal early pregnancy BMI, gestational diabetes, pre-eclampsia, chorioamnionitis, urogenital infection, high-risk pregnancy, and premature rupture of membranes. Fourth, we ran fixed effects models to compare cousins that were differentially exposed to labor induction, while adjusting for all covariates. In addition to controlling for all measured covariates, these models also controlled for all unmeasured characteristics that make cousins similar. Fifth, we ran fixed effects models to compare differentially exposed siblings. These models controlled for measured covariates unique to births and all unmeasured shared characteristics that make siblings similar. Sensitivity Analyses

We also ran five sets of sensitivity analyses to address potential bias in our methods. First, we considered whether missing maternal early pregnancy BMI information could bias the results. To address this, we estimated the population-level association in (1) a cohort of individuals that had complete information on all the covariates except maternal BMI and (2) in our main complete-case cohort, both of which adjust for all measured covariates (Model 3) except early pregnancy BMI. Second, we considered the possibility that our cohort was susceptible to left censoring due to our inability to capture diagnoses before 2001 . To address this, we assessed the association between labor induction and offspring ADHD (fully adjusted population, sibling, and cousin models) in a later-born subset of our cohort (1999-2005). Third, to address concerns about generalizability of sibling comparisons, we assessed the association between labor induction and offspring outcomes in the sub-cohort of siblings only to compare 
with our main analyses. Fourth, to specifically address any bias that may be a result of parity, effects or carry-over effects from one sibling to another, we assessed the association between labor induction and offspring outcomes in first born cousins. Fifth, we re-estimated three of the models from the main analyses (fully adjusted population, sibling, and cousin models) after excluding all deliveries by elective Caesarian to exclude mothers who would not have been atrisk of labor induction (see Oberg et al. (2016) for more details).

\section{RESULTS}

Overall, $10.3 \%$ in the cohort born between 1992 and 2005 and $8.2 \%$ in the cohort born between 1992 and 1997 were exposed to labor induction, suggesting that use of induction increased over time. Older women, first-time mothers or mothers who had already had at least three children, mothers who had higher BMI, and more pregnancy complications were more likely to be induced (see Table I and II). Mothers of siblings contributing to the estimation of the association between labor induction and ADHD had fewer years of education, were slightly younger, and were more likely to smoke during pregnancy compared with mothers in the population (see Table SI).

\section{Labor Induction to Offspring ADHD}

Kaplan-Meier estimates indicated that by the age of 21 years, $6.6 \%$ of the population overall would have been diagnosed with ADHD (if all were followed). Figures 1 and 2 plot ADHD diagnosis as a function of age, stratified by exposure to induction. Figure 1 shows the full population and Figure 2 shows differentially exposed siblings. By the age of 21 years, $6.4 \%$ of individuals unexposed to labor induction, and $8.5 \%$ of those exposed to labor induction were diagnosed with ADHD. Among differentially exposed siblings, $8.3 \%$ of the unexposed and $8.3 \%$ the exposed were diagnosed with ADHD. 
The results of our main analyses of the association between labor induction and offspring diagnosis of ADHD are reported in Table IV, and the estimation sample sizes can be found in Supplemental Table I (Table SI). Overall, offspring exposed to labor induction were at $42 \%$ increased risk of being diagnosed with ADHD compared to those not exposed (Model 1: HR $1.42,95 \%$ CI 1.38-1.46). This association was gradually attenuated by increasing degree of adjustment for measured covariates, first birth year, parity, and stable maternal characteristics (Model 2: HR 1.32, 95\% CI 1.28-1.36) and then birth-specific covariates (Model 3: HR 1.23, 95\% CI 1.19-1.28). The attenuation continued when models also controlled for unmeasured factors shared by differentially exposed cousins (Model 4: HR 1.10, 95\% CI 1.03-1.18) and finally by siblings (Model 5: HR 0.99, 95\% CI 0.91-1.07). Failure to detect an association in the fully adjusted model (which accounts for all unmeasured factors siblings share, and measured factors they may not share) suggests that labor induction is not independently associated with offspring ADHD diagnosis.

\section{Labor Induction to Offspring Academic Achievement}

We present frequency of overall grades and low test scores in math, Swedish, and English in Table III. In the population, $4.3 \%$ had low overall grades, $25.7 \%$ had low scores in math, 25.3\% had low scores in Swedish, and 30.4\% had low scores in English. The proportions were not materially different between those exposed and unexposed to labor induction or in differentially exposed siblings.

The results of our main analyses exploring the association between labor induction and offspring low academic achievement are presented in Table IV (see Table SI for sample sizes). Overall, offspring exposed to labor induction were at $15 \%$ increased odds of low grades (Model 1: HR 1.15, 95\% CI 1.10-1.21). The association was attenuated by adjustment for birth year, 
parity, stable maternal characteristics and birth-specific covariates (Model 3: OR 1.08, 95\% CI 1.02-1.14). Further adjustment for unmeasured factors shared by cousins (Model 4: OR 0.93, 95\% CI 0.76-1.13) and siblings (Model 5: OR 1.04, 95\% CI 0.88-1.32) resulted in complete attenuation of the association.

A consistent pattern of attenuation with increasing degree of adjustment was also observed in the analysis of the association between labor induction and offspring low standardized test scores (Table IV). Very weak overall associations with low test scores in math (Model 1: OR 1.03, 95\% CI 1.01-1.06) and Swedish (Model 1: OR 1.08, 95\% CI 1.06-1.08) were completely attenuated after adjustment for all measured covariates (Model 3: OR 1.02 95\% CI 0.99-1.04 and OR 0.97 95\% CI 0.94-1.00 respectively). No association to low standardized test scores in English was observed, overall or after adjustments (Model 1: OR 0.98, 95\% CI 0.95-1.00 and Model 3: OR 0.98 95\% CI 0.95-1.00).

Sensitivity Analyses

Our sensitivity analyses of the influence of data characteristics and analytic decisions are presented in Tables SIII-VII. First, we estimated models in (1) a cohort of individuals that had complete information on all the covariates except maternal BMI and (2) in our main completecase cohort, both of which adjust for all measured covariates (Model 3) except early pregnancy BMI. Between individuals in the population, labor induction was associated with offspring ADHD diagnosis, low overall grades, and low test scores in math, but not with low test scores in English and Swedish. Consistent with our main analysis, the present associations were completely attenuated in our fully adjusted cousin and sibling comparisons (see Table SIII). Second, we examined the association between labor induction and offspring ADHD in a cohort born between 1999 and 2005 (presented in Table SIV). Similar to our main analysis, labor 
induction was associated with an increased risk of offspring diagnosis of ADHD after adjustment for measured covariates, but the association was largely attenuated in the within cousins model, and was completely attenuated in the within siblings model. Third, we fit the crude and fully adjusted population models in the sub-cohort of siblings only (presented in Table SV). The association between labor induction and offspring ADHD diagnosis, low overall grades, and low test scores in math, English, and Swedish were similar to that of the main analyses, suggesting that our attenuated point estimates from the sibling-comparisons models are not due to a lower population association in the subset of the population with siblings. Fourth, we present the analyses repeated within a subset of first born cousins in Table SVI. As in our main cousin comparisons, the associations between labor induction and offspring ADHD, overall low grades, and low test scores in math, English, and Swedish were not independent of measured covariates and unmeasured factors that first born cousins share. These results imply that our main analyses were not biased due to parity and carry over effects. Fifth, we present our analysis excluding all deliveries by elective Caesarian in Table SVII. Again, the associations between labor induction and offspring ADHD, low overall grades, and low test scores in math and Swedish were present in the population (and not present in our models examining the association with low test scores in English), but were attenuated in the within cousins and siblings models.

\section{DISCUSSION}

Consistent with previous literature observing an association between labor induction and offspring neurodevelopmental problems (Gregory et al., 2013; Kurth \& Haussmann, 2011; D. Silva, L. Colvin, E. Hagemann, \& C. Bower, 2014), the current study observed increased risk of ADHD and low academic achievement for those exposed to labor induction in the population. However, we also examined the association within cousins and siblings, and found complete 
attenuation of the association, similar to the findings of Oberg et al. (2016) for the association with Autism Spectrum Disorder. This suggests that the association between labor induction and offspring ADHD diagnosis and low academic achievement is not consistent with a causal interpretation; but rather due to genetic and/or environmental factors that influence both mothers' risk of labor induction and offspring neurodevelopment. For example, low socioeconomic status is associated with medical conditions necessitating induction (e.g. gestational diabetes) (Kjos, Henry, Montoro, Buchanan, \& Mestman, 1993; Berkowitz, Lapinski, \& Lee, 1992; Nomura et al., 2012) and offspring ADHD (Nomura et al., 2012), and genetic factors are also associated with these conditions (Zhang et al., 2013; Larsson, Chang, D’Onofrio, \& Lichtenstein, 2014). In our analyses, no one covariate accounted for a substantial portion of the association between labor induction and offspring outcomes. Many of our covariates were also correlated with each other. Additional statistical approaches are needed to specify the sources of confounding, which is outside the scope of the current paper. However, given these findings, future research should specifically assess the factors that account for the association between labor induction and these outcomes in the population.

Our findings have implications for basic science, as well as health care settings. First, methodological control over both measured and unmeasured factors is important for research attempting to understand underlying biological mechanisms for the association between pregnancy-related risk factors and offspring outcomes, due to the high correlation among preand perinatal factors (D’Onofrio et al., 2016; Cammu et al., 2002; Hannah et al., 1992; Smith \& Merrill, 2006). Second, our findings, along with the findings of Oberg et al. (2016) also provide information for practitioners and patients, and may influence medical decision-making, which should weigh both the potential harms and benefits of labor induction (Hannah et al., 1992; 
Kenyon et al., 2013; Smith \& Merrill, 2006).

The current study improves upon the extant literature in this field in several ways. First, this is the largest study to examine the association between labor induction and ADHD to date and the only study we know of to examine the association between labor induction and academic achievement. Second, the quality of the Swedish registers have been well established, such that misclassification of our exposure (National Board of Health and Welfare, 2003) and outcomes (National Board of Health and Welfare, 2009; Larsson et al., 2013) is likely to be minimal. Third, we were able to adjust for many measured confounding factors that may be responsible for the observed association between labor induction and offspring ADHD and low school grades. Fourth, within cousins and siblings analyses enabled us to control for unmeasured factors that make cousins and siblings similar.

Our results must also be considered in light of several limitations. First, because we do not have measures on the method for induction, conclusions apply to the overall intervention of inducing labor. We also do not have access to information on potential labor augmentation. Hence we cannot draw conclusions about offspring exposure via oxytocin because (1) mothers who were induced may not have been exposed to oxytocin and (2) mothers who were not induced may still have been exposed to oxytocin for labor augmentation. Second, there are some limitations to using within family designs, which relate to the fact that they rely on discordance between family members (D’Onofrio et al., 2013; Frisell, Öberg, Kuja-Halkola, \& Sjölander, 2012). However, we attempted to address these limitations by using both cousin and sibling comparisons, which vary in their strengths and limitations. Further, we tested some assumptions in sensitivity analyses (obtaining similar results as our main analysis). Third, we were unable to directly assess whether genetic or environmental factors account for the association by using a 
cousin or sibling comparison. Therefore, future research should focus on this area (D'Onofrio et al., 2016). Fourth, potential concern about the extent to which these results may generalize to other populations would need to be explored by replication in other settings and countries. Specifically, prevalence rates of labor induction differ in Sweden compared to the U.S. (Coonrod, Bay, \& Kishi, 2000). However, we did use a measure of labor induction that is consistent with previous studies conducted in Sweden (Oberg et al., 2016) and the U.S. (Gregory et al., 2013). Fifth, diagnoses identified via ICD codes may be susceptible to misclassification. However, labor induction was predominantly identified from a checkbox indicator on the delivery chart, and other studies have documented the validity of ADHD diagnoses in the national patient register and the PDR (Larsson et al., 2013). Nonetheless, future studies would benefit from better measurement of both induction and neurodevelopment.

In conclusion, birth via labor induction is a predictor of offspring neurodevelopmental problems, including ADHD and low academic achievement. However, labor induction is not independently associated with these outcomes after adjustment for measured covariates and unmeasured factors shared between cousins and siblings, suggesting that labor induction itself may not cause offspring ADHD or low academic achievement. Future research should evaluate related factors that may be influencing neurodevelopmental problems, and medical professionals and pregnant women should consider these findings when contemplating labor induction.

\section{ACKNOWLEDGEMENTS}

This study was supported by grants from the Indiana Clinical and Translational Sciences Institute: Pediatric Project Development Team; the Swedish Research Council through the Swedish Initiative for Research on Microdata in the Social and Medical Sciences (SIMSAM) 
framework grant no 340-2013-5867; the National Institute of Health, T32 Grant no HD 07475.

This study was approved by the Institutional Review Board at the Karolinska Institutet.

\section{HUMAN AND ANIMAL RIGHTS AND INFORMED CONSENT}

All procedures performed in studies involving human participants were in accordance

with the ethical standards of the institutional and national research committee and/or with the

1964 Helsinki declaration and its later amendments or comparable ethical standards. For this

type of study, formal consent was not required. This article does not contain any studies with

animals performed by any of the authors.

\section{REFERENCES}

Barker, D. J. P. (1998). Mothers, babies, and health in later life: Elsevier Health Sciences.

Berkowitz, G. S., Lapinski, R. H., Wein, R., \& Lee, D. (1992). Race/ethnicity and other risk factors for gestational diabetes. American Journal of Epidemiology, 135(9), 965-973.

Bramson, L., Rickert, M., Class, Q., Sariaslan, A., Almqvist, C., Larsson, H., . . D'onofrio, B. (2016). The association between childhood relocations and subsequent risk of suicide attempt, psychiatric problems, and low academic achievement. Psychological medicine, 46(05), 969-979.

Cammu, H., Martens, G., Ruyssinck, G., \& Amy, J.-J. (2002). Outcome after elective labor induction in nulliparous women: a matched cohort study. American journal of obstetrics and gynecology, 186(2), 240-244.

Chang, Z., Lichtenstein, P., D’Onofrio, B. M., Almqvist, C., Kuja-Halkola, R., Sjölander, A., \& Larsson, H. (2014). Maternal age at childbirth and risk for ADHD in offspring: a population-based cohort study. International Journal of Epidemiology, dyu204.

Chen, Q., Sjölander, A., Långström, N., Rodriguez, A., Serlachius, E., D’Onofrio, B. M., . . . Larsson, H. (2013). Maternal pre-pregnancy body mass index and offspring attention deficit hyperactivity disorder: a population-based cohort study using a siblingcomparison design. International Journal of Epidemiology, dyt152.

Cnattingius, S., Ericson, A., Gunnarskog, J., \& Kallen, B. (1990). A quality study of a medical birth registry. Scand J Soc Med, 18(2), 143-148. Retrieved from http://www.ncbi.nlm.nih.gov/entrez/query.fcgi?cmd=Retrieve \&db=PubMed\&dopt=Citati on\&list_uids $=2367825$

Cochran, D., Fallon, D., Hill, M., \& Frazier, J. A. (2013). The role of oxytocin in psychiatric disorders: A review of biological and therapeutic research findings. Harvard review of psychiatry, 21(5), 219.

D’Onofrio B.M., Class Q.A., Rickert M.E., et al. Translational epidemiologic approaches to understanding the consequences of early-life exposures. Behavior genetics. 2016;46(3):315-328. 
D’Onofrio, B. M., Lahey, B. B., Turkheimer, E., \& Lichtenstein, P. (2013). The critical need for family-based, quasi-experimental research in integrating genetic and social science research. American Journal of Public Health, 103, S46-S55.

D'onofrio, B. M., Singh, A. L., Iliadou, A., Lambe, M., Hultman, C. M., Grann, M., .. . Lichtenstein, P. (2010). Familial confounding of the association between maternal smoking during pregnancy and offspring criminality: a population-based study in Sweden. Archives of general psychiatry, 67(5), 529-538.

Frost, F., Starzyk, P., George, S., \& McLaughlin, J. F. (1984). Birth complication reporting: the effect of birth certificate design. American journal of public health, 74(5), 505-506.

Frisell, T., Öberg, S., Kuja-Halkola, R., \& Sjölander, A. (2012). Sibling comparison designs: bias from non-shared confounders and measurement error. Epidemiology, 23(5), 713-720.

Getahun, D., Rhoads, G. G., Demissie, K., Lu, S. E., Quinn, V. P., Fassett, M. J., ... \& Jacobsen, S. J. (2013). In utero exposure to ischemic-hypoxic conditions and attentiondeficit/hyperactivity disorder. Pediatrics, 131(1), e53-e61.

Gregory, S. G., Anthopolos, R., Osgood, C. E., Grotegut, C. A., \& Miranda, M. L. (2013). Association of autism with induced or augmented childbirth in North Carolina Birth Record (1990-1998) and Education Research (1997-2007) databases. JAMA Pediatr, 167(10), 959-966. doi:10.1001/jamapediatrics.2013.2904

Hannah, M. E., Hannah, W. J., Hellmann, J., Hewson, S., Milner, R., \& Willan, A. (1992). Induction of labor as compared with serial antenatal monitoring in post-term pregnancy: a randomized controlled trial. New England Journal of Medicine, 326(24), 1587-1592.

Heckman, J. J. (2012). The developmental origins of health. Health Econ, 21(1), 24-29. doi: $10.1002 /$ hec. 1802

Henderson, C. (2000). Oxytocin gene necessary for social memory. Pain Central Nervous System Week.

Hiby, S. E., Walker, J. J., O'Shaughnessy, K. M., Redman, C. W., Carrington, M., Trowsdale, J., \& Moffett, A. (2004). Combinations of maternal KIR and fetal HLA-C genes influence the risk of preeclampsia and reproductive success. Journal of Experimental Medicine, 200(8), 957-965.

Kenyon, S., Tokumasu, H., Dowswell, T., Pledge, D., \& Mori, R. (2013). High- dose versus low- dose oxytocin for augmentation of delayed labour. The Cochrane Library.

Kjos, S. L., Henry, O. A., Montoro, M., Buchanan, T. A., \& Mestman, J. H. (1993). Insulinrequiring diabetes in pregnancy: a randomized trial of active induction of labor and expectant management. American journal of obstetrics and gynecology, 169(3), 611-615.

Kurth, L., \& Haussmann, R. (2011). Perinatal Pitocin as an early ADHD biomarker: neurodevelopmental risk? Journal of attention disorders, 1087054710397800.

Lambe, M., Hultman, C., Torrång, A., MacCabe, J., \& Cnattingius, S. (2006). Maternal smoking during pregnancy and school performance at age 15. Epidemiology, 17(5), 524-530.

Larsson, H., Chang, Z., D'Onofrio, B. M., \& Lichtenstein, P. (2014). The heritability of clinically diagnosed attention deficit hyperactivity disorder across the lifespan. Psychological medicine, 44(10), 2223-2229.

Larsson, H., Rydén, E., Boman, M., Långström, N., Lichtenstein, P., \& Landén, M. (2013). Risk of bipolar disorder and schizophrenia in relatives of people with attention-deficit hyperactivity disorder. The British Journal of Psychiatry, 203(2), 103-106. 
Larsson, H., Sariaslan, A., Långström, N., D'Onofrio, B., \& Lichtenstein, P. (2014). Family income in early childhood and subsequent attention deficit/hyperactivity disorder: a quasi- experimental study. Journal of Child Psychology and Psychiatry, 55(5), 428-435.

Ludvigsson, J. F., Otterblad-Olausson, P., Pettersson, B. U., \& Ekbom, A. (2009). The Swedish personal identity number: possibilities and pitfalls in healthcare and medical research. Eur J Epidemiol, 24(11), 659-667. doi:10.1007/s10654-009-9350-y

Luthy, D. A., Malmgren, J. A., \& Zingheim, R. W. (2004). Cesarean delivery after elective induction in nulliparous women: the physician effect. American journal of obstetrics and gynecology, 191(5), 1511-1515.

Nassar, A. H., Adra, A. M., Chakhtoura, N., Gómez-Marín, O., \& Beydoun, S. (1998). Severe preeclampsia remote from term: labor induction or elective cesarean delivery? American journal of obstetrics and gynecology, 179(5), 1210-1213.

National Board of Health and Welfare. (2003). The Swedish Medical Birth Register - A Summary of Content and Quality. Retrieved from Stockholm:

National Board of Health and Welfare. (2009). Quality and Content in the Patient Register Inpatient care discharges 1964-2007 and outpatient (specialized care) visits 1997-2007 [in Swedish] (2009-125-15). Retrieved from Stockholm:

Nomura, Y., Marks, D. J., Grossman, B., Yoon, M., Loudon, H., Stone, J., \& Halperin, J. M. (2012). Exposure to gestational diabetes mellitus and low socioeconomic status: effects on neurocognitive development and risk of attention-deficit/hyperactivity disorder in offspring. Archives of pediatrics \& adolescent medicine, 166(4), 337-343.

Oberg, A. S., D’Onofrio, B. M., Rickert, M. E., Hernandez-Diaz, S., Ecker, J. L., Almqvist, C., . .. Bateman, B. T. (2016). Association of Labor Induction With Offspring Risk of Autism Spectrum Disorders. JAMA Pediatr, e160965-e160965.

Osterman, M. J., \& Martin, J. A. (2014). Recent declines in induction of labor by gestational age. NCHS data brief, 155(155), 1-8. Retrieved from http://www.ncbi.nlm.nih.gov/pubmed/24941926

Saade, G. R., Sibai, B. M., \& Silver, R. (2014). Induction or augmentation of labor and autism. JAMA Pediatr, 168(2), 190-191. doi:10.1001/jamapediatrics.2013.4800

Silva, D., Colvin, L., Hagemann, E., \& Bower, C. (2014). Environmental risk factors by gender associated with attention-deficit/hyperactivity disorder. Pediatrics, 133(1), e14-e22.

Silva, D., Colvin, L., Hagemann, E., \& Bower, C. (2014). Environmental risk factors by gender associated with attention-deficit/hyperactivity disorder. Pediatrics, 133(1), e14-22. doi:10.1542/peds.2013-1434

Skoglund, C., Chen, Q., Lichtenstein, P., \& Larsson, H. (2014). Familial confounding of the association between maternal smoking during pregnancy and ADHD in offspring. Journal of Child Psychology and Psychiatry, 55(1), 61-68.

Smith, J. G., \& Merrill, D. C. (2006). Oxytocin for induction of labor. Clinical obstetrics and gynecology, 49(3), 594-608.

Spong, C. Y., Landon, M. B., Gilbert, S., Rouse, D. J., Leveno, K. J., Varner, M. W., . . . Wapner, R. J. (2007). Risk of uterine rupture and adverse perinatal outcome at term after cesarean delivery. Obstetrics \& Gynecology, 110(4), 801-807.

Statistics Sweden. (2010). Multi-Generation Register 2009 - A Description of Contents and Quality (2010:13). Retrieved from Örebro, Sweden:

Stitely, M. L. (2014). Induction or augmentation of labor and autism. JAMA Pediatr, 168(2), 189-190. doi:10.1001/jamapediatrics.2013.4789 
Vintzileos, A., \& Ananth, C. Induction or augmentation of labor and autism. JAMA Pediatr 2014; 1168: 190.@ 2014 Mosby, Inc. All rights reserved.

Vivian- Taylor, J., Sheng, J., Hadfield, R. M., Morris, J. M., Bowen, J. R., \& Roberts, C. L. (2011). Trends in obstetric practices and meconium aspiration syndrome: a populationbased study. BJOG: An International Journal of Obstetrics \& Gynaecology, 118(13), 1601-1607.

Wahl, R. U. (2004). Could oxytocin administration during labor contribute to autism and related behavioral disorders?--A look at the literature. Med Hypotheses, 63(3), 456-460. doi:10.1016/j.mehy.2004.03.008

Walker, C. K., Krakowiak, P., Baker, A., Hansen, R. L., Ozonoff, S., \& Hertz-Picciotto, I. (2015). Preeclampsia, placental insufficiency, and autism spectrum disorder or developmental delay. JAMA pediatrics, 169(2), 154-162.

WHO. (1992). International Classification of Diseases, World Health Organization WHO. Geneva.

WHO. (2004). International Statistical Classification of Diseases and Health Related Problems (The) ICD-10. World Health Organization.

Winslow, J. T., \& Insel, T. R. (2002). The social deficits of the oxytocin knockout mouse. Neuropeptides, 36(2), 221-229.

Zhang, C., Bao, W., Rong, Y., Yang, H., Bowers, K., Yeung, E., \& Kiely, M. (2013). Genetic variants and the risk of gestational diabetes mellitus: a systematic review. Human reproduction update, 19(4), 376-390. 
Figure 1. Relationship between labor induction and attention-deficit hyperactivity disorder in the population

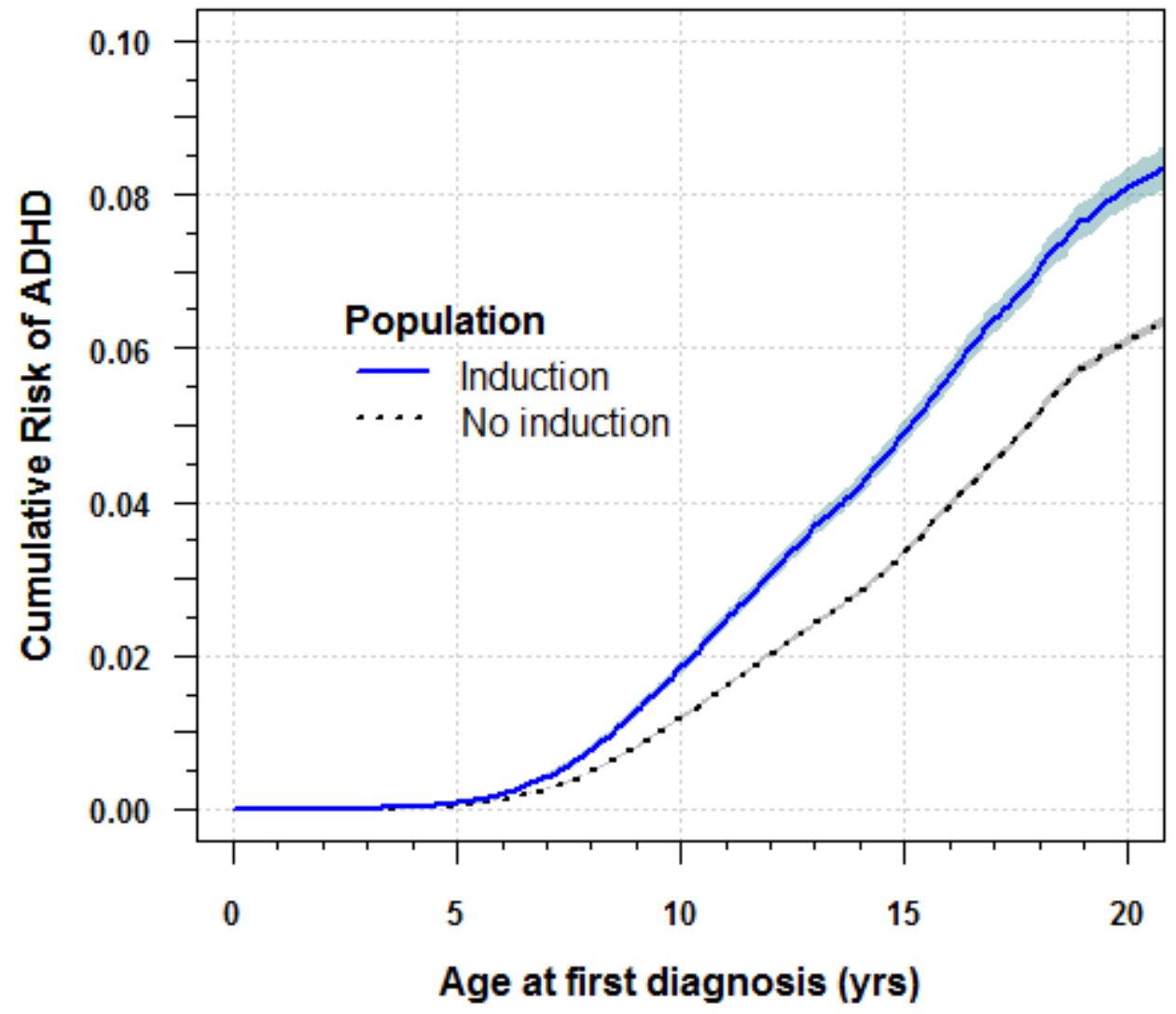

Cumulative risk of being diagnosed with ADHD as a function of age. The shaded areas around the curve represent the $95 \%$ CI on the failure proportion. 
Figure 2 .Relationship between labor induction and attention-deficit hyperactivity disorder in differentially exposed siblings

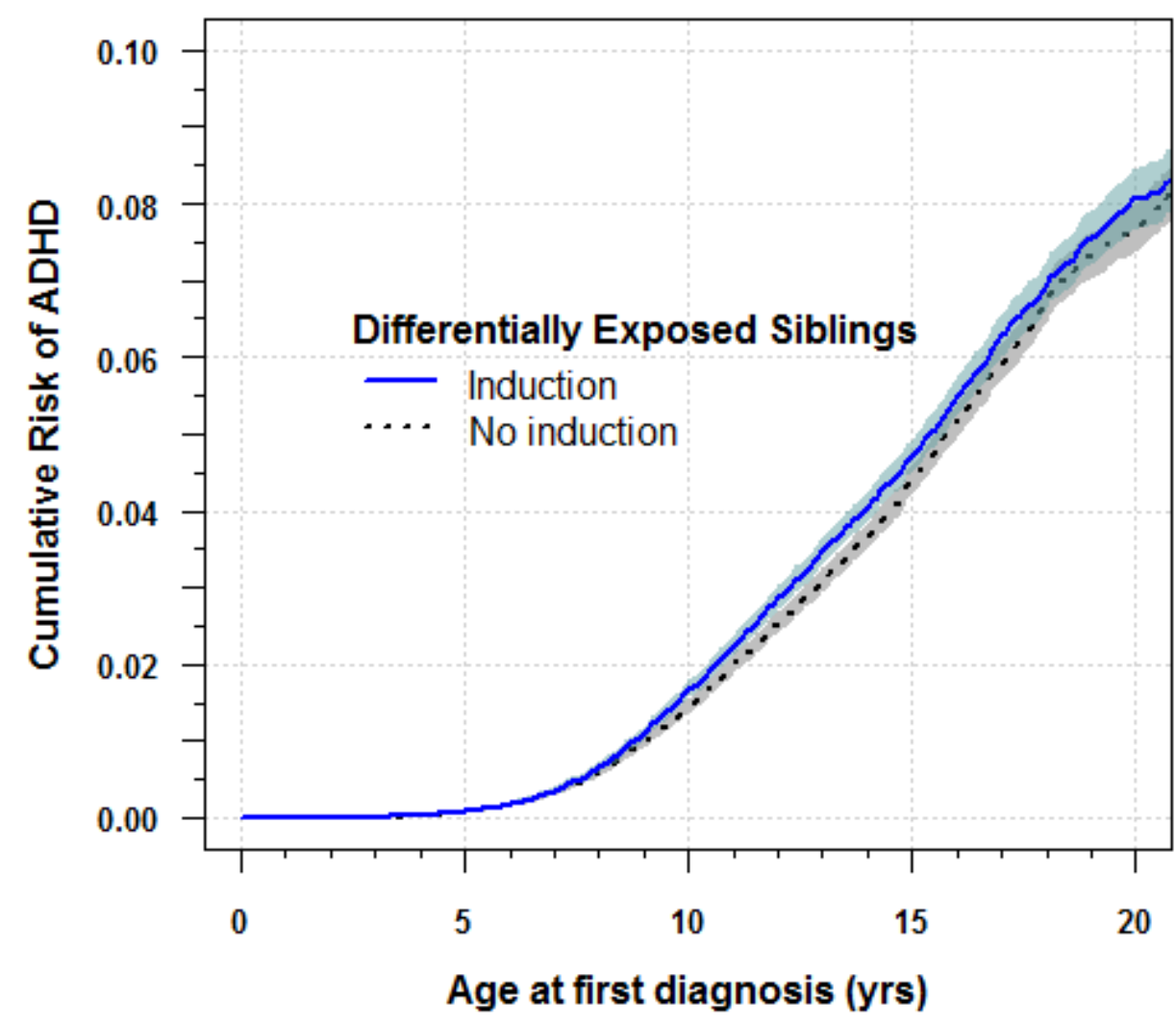

Cumulative risk of being diagnosed with ADHD as a function of age. The shaded areas around the curve represent the $95 \% \mathrm{CI}$ on the failure proportion. 
Table I. Maternal and pregnancy characteristics frequencies for the n=1,085,008 live births 1992-2005

\section{Maternal Characteristics}

Highest Education

Country of birth

\section{married/co-hat
living alone}

\section{Pregnancy characteristics}

Age at delivery

$\begin{aligned} & 20-29 \\ & 30-39 \\ & \text { over } 40 \\ & \text { Birth Year } \begin{array}{l}1992-1997 \\ 1998-2005\end{array} \\ & \text { Parity } 1 \\ & 2 \\ & 3 \\ & 4 \text { or more }\end{aligned}$

\begin{tabular}{ccc} 
All & No induction & Induction \\
\hline $\mathrm{n}(\%)$ & $\mathrm{n}(\%)$ & $\mathrm{n}(\%)$ \\
$17678(1.63)$ & $15970(1.64)$ & $1708(1.53)$ \\
$75215(6.93)$ & $66957(6.88)$ & $8258(7.42)$ \\
$514218(47.39)$ & $460936(47.34)$ & $53282(47.86)$ \\
$477897(44.05)$ & $429809(44.14)$ & $48088(43.19)$ \\
& & \\
$911374(84.00)$ & $817258(83.94)$ & $94116(84.53)$ \\
$173634(16.00)$ & $156414(16.06)$ & $17220(15.47)$ \\
& & \\
$1032380(95.15)$ & $927250(95.23)$ & $105130(94.43)$ \\
$52628(4.85)$ & $46422(4.77)$ & $6206(5.57)$
\end{tabular}

$20621(1.90) \quad 18835(1.93) \quad 1786(1.60)$

$555121(51.16) \quad 503508(51.71) \quad 51613(46.36)$

$483792(44.59) \quad 429698(44.13) \quad 54094(48.59)$

$25474(2.35) \quad 21631(2.22) \quad 3843(3.45)$

$489196(45.09) \quad 449084(46.12) \quad 40112(36.03)$

$595812(54.91) \quad 524588(53.88) \quad 71224(63.97)$

$459854(42.38) \quad 406871(41.79) \quad 52983(47.59)$

$400068(36.87) \quad 367907(37.79) \quad 32161(28.89)$

$157297(14.50) \quad 140475(14.43) \quad 16822(15.11)$

$67789(6.25) \quad 58419(6.00) \quad 9370(8.42)$ 
Early Pregnancy BMI <18.5

$18.5-24$

25-29

$30-34$

$>35$

Smoking No smoking

1-10/day

$\geq 10 /$ day

Gestational diabetes

Gestational hypertension

Pre-eclampsia

Chorioamniontis

Urogenital infection

High-risk pregnancy

Premature rupture of membranes

Post-term gestation

IUGR (ICD diagnosis)

$\begin{array}{ccc}29206(2.69) & 27139(2.79) & 2067(1.86) \\ 712791(65.69) & 649907(66.75) & 62884(56.48) \\ 251922(23.22) & 220874(22.68) & 31048(27.89) \\ 71565(6.60) & 59986(6.16) & 11579(10.40) \\ 19524(1.80) & 15766(1.62) & 3758(3.38) \\ & & \\ 931905(85.89) & 836243(85.89) & 95662(85.92) \\ 102016(9.40) & 91563(9.40) & 10453(9.39) \\ 51087(4.71) & 45866(4.71) & 5221(4.69) \\ & & \\ 7357(0.68) & 5495(0.56) & 1862(1.67) \\ 9552(0.88) & 6426(0.66) & 3126(2.81) \\ 31493(2.90) & 14833(1.52) & 16660(14.96) \\ 2045(0.19) & 1535(0.16) & 510(0.46) \\ 125379(11.56) & 110825(11.38) & 14554(13.07) \\ 3193(0.29) & 2262(0.23) & 931(0.84) \\ 13426(1.24) & 10987(1.13) & 2439(2.19) \\ 66490(6.13) & 38879(3.99) & 27611(24.80) \\ 28538(2.63) & 20990(2.16) & 7548(6.78) \\ 527813(48.65) & 477733(49.07) & 50080(44.98)\end{array}$


Table II. Associations between labor induction and maternal and pregnancy characteristics for the n=1,085,008 live births 1992-2005

\begin{tabular}{|c|c|c|}
\hline \multicolumn{2}{|l|}{ Maternal Characteristics } & OR $(95 \% \mathrm{CI})$ \\
\hline \multirow[t]{4}{*}{ Highest Education } & less than 9 years & -- \\
\hline & completed 9 years & $1.19(1.14,1.25)$ \\
\hline & $\begin{array}{l}\text { upper secondary 1-3 } \\
\text { years }\end{array}$ & $1.12(1.08,1.17)$ \\
\hline & post-secondary (any) & $1.10(1.06,1.15)$ \\
\hline \multirow[t]{2}{*}{ Country of birth } & Sweden & $1.06(1.05,1.08)$ \\
\hline & Other & -- \\
\hline \multirow[t]{2}{*}{ Civil Status } & married/co-habitating & -- \\
\hline & living alone & $1.15(1.13,1.18)$ \\
\hline \multicolumn{3}{|l|}{ Pregnancy characteristics } \\
\hline & less than 20 & $0.90(0.86,0.94)$ \\
\hline \multirow[t]{3}{*}{ Age at delivery } & $20-29$ & -- \\
\hline & $30-39$ & $1.24(1.23,1.26)$ \\
\hline & over 40 & $1.71(1.66,1.76)$ \\
\hline \multirow[t]{2}{*}{ Birth Year } & 1992-1997 & -- \\
\hline & $1998-2005$ & $1.52(1.50,1.54)$ \\
\hline \multirow[t]{4}{*}{ Parity } & 1 & -- \\
\hline & 2 & $0.70(0.69,0.71)$ \\
\hline & 3 & $0.95(0.94,0.97)$ \\
\hline & 4 or more & $1.26(1.24,1.29)$ \\
\hline Early Pregnancy BMI & $<18.5$ & $0.79(0.76,0.82)$ \\
\hline
\end{tabular}


$18.5-24$

25-29

30-34

$>35$

Smoking No smoking

1-10/day

$\geq 10 /$ day

Gestational diabetes

Gestational hypertension

Pre-eclampsia

Chorioamniontis

Urogenital infection

High-risk pregnancy

Premature rupture of membranes

Post-term gestation

IUGR (ICD diagnosis)
$1.45(1.43,1.47)$

$1.95(1.91,1.99)$

$2.40(2.31,2.48)$

$1.00(0.98,1.02)$

$0.99(0.96,1.02)$

$2.90(2.77,3.04)$

$4.13(3.97,4.29)$

$10.22(10.02,10.42)$

$2.64(2.42,2.88)$

$1.17(1.15,1.19)$

$3.20(2.99,3.43)$

$1.59(1.53,1.65)$

$7.40(7.29,7.51)$

$3.21(3.14,3.29)$

$0.86(0.85,0.87)$ 
Table III. Low academic achievement frequencies for $n=489,196$ live births 1992-1997

\begin{tabular}{lccc}
\hline Academic Variable & All & No induction & Induction \\
\hline Population & $\mathrm{n}(\%)$ & $\mathrm{n}(\%)$ & $\mathrm{n}(\%)$ \\
\cline { 2 - 4 } Overall low grades & $19984(4.31)$ & $18147(4.26)$ & $1837(4.87)$ \\
$\quad$ Low math scores & $119055(25.65)$ & $109081(25.58)$ & $9974(26.43)$ \\
$\quad$ Low Swedish scores & $117346(25.28)$ & $107199(25.14)$ & $10147(26.89)$ \\
$\quad$ Low English scores & $141021(30.38)$ & $129654(30.40)$ & $11367(30.12)$ \\
Differentially Exposed Siblings & & & \\
$\quad$ Overall low grades & $10932(4.08)$ & $9969(4.03)$ & $963(4.61)$ \\
$\quad$ Low math scores & $65012(24.24)$ & $59728(24.15)$ & $5284(25.30)$ \\
$\quad$ Low English scores & $80636(30.07)$ & $74370(30.08)$ & $6266(30.00)$ \\
$\quad$ Low Swedish scores & $69551(25.94)$ & $63776(25.79)$ & $5775(27.65)$ \\
\hline
\end{tabular}


Table IV. Point estimates (95\% CI) of the association between labor induction and offspring ADHD and low school grades (completecase)

\begin{tabular}{lccccc}
\hline \multicolumn{1}{c}{ Outcome } & Model 1 & Model 2 & Model 3 & Model 4 & Model 5 \\
\hline ADHD $^{\mathbf{a}}$ & $1.42(1.38,1.46)$ & $1.32(1.28,1.36)$ & $1.23(1.19,1.28)$ & $1.10(1.03,1.18)$ & $0.99(0.91,1.07)$ \\
Overall low grades $^{\mathbf{b}}$ & $1.15(1.10,1.21)$ & $1.14(1.08,1.20)$ & $1.08(1.02,1.14)$ & $0.93(0.76,1.13)$ & $1.04(0.88,1.22)$ \\
Low math score $^{\mathbf{b}}$ & $1.03(1.01,1.06)$ & $1.06(1.03,1.08)$ & $1.02(0.99,1.04)$ & $1.01(0.92,1.10)$ & $1.00(0.93,1.07)$ \\
Low English score $^{\mathbf{b}}$ & $0.98(0.95,1.00)$ & $1.02(0.99,1.04)$ & $0.98(0.95,1.00$ & $0.90(0.80,1.02)$ & $1.01(0.94,1.08)$ \\
Low Swedish score $^{\mathbf{b}}$ & $1.08(1.06,1.11)$ & $1.03(1.01,1.06)$ & $0.97(0.94,1.00)$ & $0.95(0.85,1.05)$ & $0.94(0.87,1.02)$ \\
\hline a & & & & &
\end{tabular}

${ }^{a}$ hazard ratio

bodds ratio

Model 1: Population estimate without covariate adjustment

Model 2: Population estimate with adjustment for birth year, parity, maternal age at birth, maternal education, maternal civil status, and maternal country of origin

Model 3: Population estimate with additional adjustment for smoking and BMI in early pregnancy, gestational diabetes or hypertension, pre-eclampsia, chorioamnionitis, urogenital infection, inter-uterine growth restriction, premature rupture of membranes, post-term gestation, high-risk pregnancy, and offspring sex

Model 4: Within cousins estimate including adjustment for all measured covariates

M5: Within siblings estimate including adjustment for birth year, parity, and all other birth- specific factors (from Model 3) 


\section{SUPPLEMENTAL TABLES AND RESULTS}

Table SI. Maternal and pregnancy characteristics frequencies among siblings

\section{Maternal Characteristics}

Highest Education

Country of birth

Civil Status

\section{Pregnancy characteristics}

Age at delivery

Birth Year

Parity

\begin{tabular}{c}
\hline Siblings \\
\hline $\mathrm{n}(\%)$ \\
$411(1.14)$ \\
$4913(13.64)$ \\
$20178(56.04)$ \\
$10502(29.16)$ \\
$32145(89.28)$ \\
$3859(10.72)$
\end{tabular}

33062 (91.83)

$2942(8.17)$

$$
\begin{gathered}
1480(4.11) \\
22494(62.48) \\
11612(32.25) \\
418(1.16)
\end{gathered}
$$

$19221(53.39)$

16783 (46.61)

$12195(33.87)$

14441 (40.11)

$5912(16.42)$ 
4 or more

$<18.5$

18.5-24

25-29

30-34

$>35$

Smoking

$$
3456 \text { (9.60) }
$$

$1044(2.90)$

20603 (57.22)

9205 (25.57)

3584 (9.95)

1568 (4.36)

26145 (72.62)

$5900(16.39)$

3959 (11.00)

Gestational diabetes

$$
\begin{gathered}
285(0.79) \\
265(0.74) \\
1069(2.97) \\
69(0.20) \\
4583(12.73) \\
100(0.28) \\
506(1.41) \\
1914(5.32) \\
1107(3.07) \\
14187(39.40)
\end{gathered}
$$

Note: We identified cousins and siblings that were discordant on ADHD, as any cousins or siblings that did not have any variance in ADHD diagnosis would not contribute to the estimate of the association between labor induction and offspring ADHD. 
SII. Estimation sample sizes for modeling results presented in Table III

\begin{tabular}{lccccc}
\hline Outcome & Model 1 & Model 2 & Model 3 & Model 4 & Model 5 \\
\hline ADHD & $1085008^{\mathrm{a}}$ & $1085008^{\mathrm{a}}$ & $1085008^{\mathrm{a}}$ & $51931^{\mathrm{b}}$ & $64762^{\mathrm{c}}$ \\
Overall low grades & $464175^{\mathrm{d}}$ & $464175^{\mathrm{d}}$ & $464175^{\mathrm{d}}$ & $9949^{\mathrm{e}}$ & $12129^{\mathrm{f}}$ \\
Low math score & $489196^{\mathrm{g}}$ & $489196^{\mathrm{g}}$ & $489196^{\mathrm{g}}$ & $9949^{\mathrm{e}}$ & $12129^{\mathrm{f}}$ \\
Low English score $^{\mathrm{b}}$ & $489196^{\mathrm{g}}$ & $489196^{\mathrm{g}}$ & $489196^{\mathrm{g}}$ & $9949^{\mathrm{e}}$ & $12129^{\mathrm{f}}$ \\
Low Swedish score $^{\mathrm{b}}$ & $489196^{\mathrm{g}}$ & $489196^{\mathrm{g}}$ & $489196^{\mathrm{g}}$ & $9949^{\mathrm{e}}$ & $12129^{\mathrm{f}}$ \\
\hline
\end{tabular}

${ }^{\mathrm{a}} \mathrm{N}$ for Cohort born between 1991 and 2005

${ }^{b} \mathrm{~N}$ for Differentially exposed cousins born between 1991 and 2005

${ }^{\mathrm{c}} \mathrm{N}$ for Differentially exposed siblings born between 1991 and 2005

${ }^{\mathrm{d}} \mathrm{N}$ for Cohort born between 1991 and 1997 (with no missing on overall low grades)

${ }^{\mathrm{e}} \mathrm{N}$ for Differentially exposed cousins born between 1991 and 1997

${ }^{\mathrm{f}} \mathrm{N}$ for Differentially exposed siblings born between 1991 and 1997

${ }^{\mathrm{g}} \mathrm{N}$ for Cohort born between 1991 and 1997 (with no missing on low test scores in math, English, and Swedish) 
SIII. Table SII. Point estimates (95\% CI) of the fully adjusted association between labor induction and offspring ADHD and low school grades without adjustment for early pregnancy BMI (complete-case)

\begin{tabular}{lcc}
\hline \multicolumn{1}{c}{ Outcome } & Missing on BMI only & Complete-Case \\
\hline ADHD $^{\mathbf{a}}$ & $1.25(1.21,1.29)$ & $1.28(1.23,1.32)$ \\
Low grades $^{\mathbf{b}}$ & $1.10(1.04,1.15)$ & $1.10(1.04,1.17)$ \\
Low math score $^{\mathrm{b}}$ & $1.04(1.01,1.06)$ & $1.03(1.01,1.06)$ \\
Low English score $^{\mathrm{b}}$ & $0.99(0.97,1.02)$ & $1.01(0.99,1.04)$ \\
Low Swedish score $^{\mathrm{b}}$ & $0.97(0.95,1.00)$ & $0.98(0.96,1.01)$ \\
\hline${ }^{\text {ahazard ratio }}$ & &
\end{tabular}

hazard ratio

Consistent with our fully adjusted population models in the main analyses, after adjustment for all variables except maternal BMI, labor induction was associated with offspring ADHD diagnosis (HR 1.25 95\% CI 1.21-1.29), low overall grades (OR 1.10 95\% CI 1.04-1.15), low test scores in math (OR $1.0495 \%$ CI 1.01-1.06), but not associated with low test scores in English (OR 0.99 95\% CI 0.97-1.02) or Swedish (OR 0.97 95\% CI 0.95-1.00) in a cohort of individuals that had complete information on all covariates with the exception of maternal BMI. Additionally, labor induction was associated with offspring ADHD (HR 1.28 95\% CI 1.23-1.32), low overall grades (OR 1.10 95\% CI 1.04-1.17), low test scores in math (OR 1.03 95\% CI 1.01-1.06), but not low test scores in English (OR 1.01 95\% CI 0.99-1.04) or Swedish (OR 0.98 95\% CI 0.96-1.01) in the main complete-case cohort. This suggests that our analytic decision to drop individuals without complete information is not biasing the point estimates observed in the original models. 
SIIIV. Point estimates (95\% CI) of the fully adjusted association between labor induction and offspring ADHD in cohort born between 1999 and 2005(complete-case)

\begin{tabular}{lccc}
\hline Outcome & Model 3 & Model 4 & Model 5 \\
ADHD & $1.21(1.15,1.27)$ & $1.08(0.94,1.23)$ & $0.86(0.76,0.98)$ \\
\hline
\end{tabular}

Because this cohort was born 1999-2005, we were only able to run analyses examining the association between labor induction and offspring ADHD diagnosis. Again, similar to the main analyses, labor induction was associated with a $21 \%$ increased risk of offspring being diagnosed with ADHD (Model 3). However, this association was attenuated within cousins (Model 4HR $1.0895 \%$ CI 0.94-1.23) and siblings (Model 5 HR 0.86 95\% CI 0.76-0.98), which also lends support to the main findings. 
SIV. Point estimates (95\% CI) of the crude (Model 1) and fully adjusted (Model 3) associations between labor induction and offspring ADHD and low school grades in siblings only

\begin{tabular}{lcc}
\hline \multicolumn{1}{c}{ Outcome } & Model 1 & Model 3 \\
\hline ADHD $^{\mathbf{a}}$ & $1.45(1.39,1.52)$ & $1.26(1.20,1.32)$ \\
Low overall grades $^{\mathbf{b}}$ & $1.16(1.07,1.25)$ & $1.07(0.98,1.16)$ \\
Low math score $^{\mathbf{b}}$ & $1.03(1.00,1.07)$ & $0.98(0.94,1.02)$ \\
Low English score $^{\mathbf{b}}$ & $1.00(0.95,1.02)$ & $0.96(0.92,1.00)$ \\
Low Swedish score $^{\mathbf{b}}$ & $1.08(1.04,1.12)$ & $1.05(1.01,1.09)$ \\
\hline hazard ratio $^{\mathbf{a}}$ & & \\
odds ratio $^{\mathbf{b}}$ & &
\end{tabular}

The associations between labor induction and offspring ADHD diagnosis (HR 1.45 95\% CI 1.39-1.52), low overall grades (OR $1.1695 \%$ CI 1.07-1.25), and low test scores in math (OR 1.03 95\% CI 1.00-1.07) and Swedish (OR 1.08 95\% CI 1.04-1.12) were all present in the sample of siblings only (Model 1), and an association with low test scores in English was not present (OR 1.00 95\% CI 0.95-1.02). However, adjustment for measured covariates led to a partial attenuation of the associations to offspring ADHD diagnosis (HR 1.26 95\% CI 1.20-1.32) and low test scores in Swedish (OR 1.05 95\% CI 1.01-1.09), and full attenuation of the association to low overall grades (OR 1.07 95\% CI 0.98-1.16) and low test scores in math (OR $0.9895 \%$ CI 0.94-1.02), consistent with the main analyses. This suggests that our results were not strongly biased by restriction to siblings. 
SVI. Point estimates (95\% CI) of the fully adjusted association between labor induction and offspring ADHD and low school grades within first born cousins (complete-case)

\begin{tabular}{lc}
\hline \multicolumn{1}{c}{ Outcome } & Model 4 \\
\hline ADHD $^{\mathbf{a}}$ & $1.10(0.89,1.36)$ \\
Overall low grades $^{\mathbf{b}}$ & $1.03(0.90,1.16)$ \\
Low math score $^{\mathrm{b}}$ & $1.02(0.96,1.08)$ \\
Low English score $^{\mathbf{b}}$ & $0.97(0.91,1.02)$ \\
Low Swedish score $^{\mathbf{b}}$ & $0.94(0.88,1.00)$ \\
\hline hazard ratio $^{\mathbf{a}}$ & \\
odds ratio $^{\mathbf{b}}$ &
\end{tabular}

As in our main cousin and sibling comparisons, the associations between labor induction and offspring ADHD (HR 1.10 95\% CI 0.89-1.36), overall low grades (OR 1.03 95\% CI 0.90-1.16), and low test scores in math (OR 1.02 95\% CI 0.96-1.08), English (OR $0.9795 \%$ CI 0.91-1.02) and Swedish (OR 0.94 95\% CI 0.88-1.00) were not independent of measured covariates and unmeasured factors that first born cousins share. This suggests that parity and carry over effects were unlikely to have a great influence on our findings. 
SVII. Fully adjusted association between labor induction and offspring ADHD and low school grades excluding deliveries by elective Caesarian (complete-case)

\begin{tabular}{lccc}
\hline \multicolumn{1}{c}{ Outcome } & Model 3 & Model 4 & Model 5 \\
\hline ADHD $^{\text {a }}$ & $1.30(1.26,1.35)$ & $1.15(1.06,1.25)$ & $1.02(0.93,1.12)$ \\
Overall low grades $^{\mathbf{b}}$ & $1.16(1.07,1.25)$ & $1.05(0.84,1.31)$ & $1.07(0.98,1.16)$ \\
Low math score $^{\mathbf{b}}$ & $1.03(1.00,1.07)$ & $1.01(0.91,1.12)$ & $0.98(0.94,1.02)$ \\
Low English score $^{\mathbf{b}}$ & $1.00(0.97,1.02)$ & $0.91(0.80,1.04)$ & $0.98(0.91,1.05)$ \\
Low Swedish score $^{\mathbf{b}}$ & $1.08(1.04,1.12)$ & $0.97(0.86,1.10)$ & $1.05(1.01,1.09)$ \\
\hline hazard ratio $^{\mathbf{a}}$ & & & \\
odds ratio $^{\mathbf{b}}$ & & &
\end{tabular}

Again, the associations between labor induction and offspring ADHD (HR 1.30 95\% CI 1.26-1.35), low overall grades (OR $1.1695 \%$ CI 1.07-1.25), and low test scores in math (OR 1.03 95\% CI 1.00-1.07), and Swedish (OR 1.08 95\% CI 1.04-1.12) were present in the population (Model 3), but were attenuated in the within cousins (Model 4) and siblings (Model 5) models (see Table 7). The association between labor induction and offspring low test scores in English was not present in the population (OR $1.0095 \%$ CI 0.97-1.02) or after adjustment (see Table 7). These effect sizes are similar to those found in the fully adjusted population model in our main analyses, suggesting that including deliveries by elective Caesarian in our main analyses did not strongly influence our findings. 